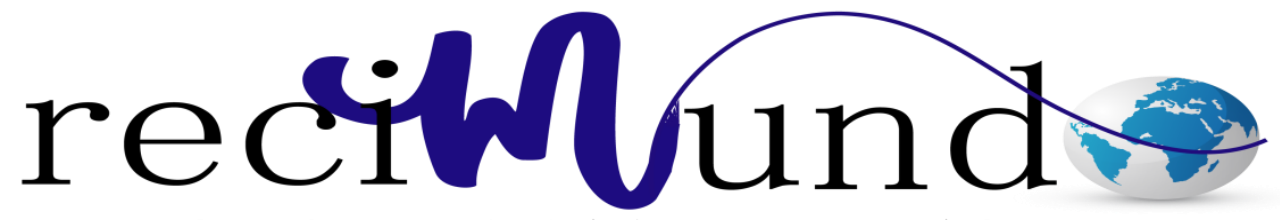

Revista Cientifica Mundo de la Investigación y el Conocimiento

Paulino Javier Suarez Guamán ${ }^{\text {a }}$; María Pastora Guamán Sánchez 하 Virginia Johanna Suarez Guamán ${ }^{c}$; Kerly Pastora Suarez Guamán ${ }^{\text {d }}$

La educación superior en el Ecuador Vs Las nuevas tecnologías

Revista Científica Mundo de la Investigación y el Conocimiento. Vol. 1 núm., 5, diciembre, 2017, pp. 484-496

DOI: $10.26820 /$ recimundo/1.5.2017.484-496

Editorial Saberes del Conocimiento 


\section{La educación superior en el Ecuador Vs Las nuevas tecnologías}

Vol. 1, núm. 5., (2017)

Paulino Javier Suarez Guamán; María Pastora Guamán Sánchez; Virginia Johanna Suarez

Guamán; Kerly Pastora Suarez Guamán

\section{RESUMEN}

La llegada del internet a todos los ámbitos de la sociedad no ha escapado al entorno educativo, ya desde hace muchos años se ha incorporado la educación a distancia por medio de universidades virtuales, que son un preámbulo de lo que nos depara el futuro entorno a las tecnología de información y su aplicación en los entornos educativos a nivel de universidades y otros estudios de alto nivel. La metodología empleada en la presente investigación es una revisión bibliográfica documental de tipo no experimental. En vista que se utilizó como método de investigación diferentes artículos y trabajos de investigación alojados en páginas web, referente a la temática de la educación superior en el Ecuador y si han habido avances en materia tecnológica para mejorarla. Algunas conclusiones a las que se pueden llegar es que con el cambio de poder en el Ecuador en el año 2007 se logró institucionalizar la temática sobre las nuevas tecnologías en el entorno educativo a nivel superior en el Ecuador con la creación de la Secretaria de Educación Superior, Ciencia, Tecnología e Innovación (SENASCYT), la Secretaría Nacional de Planificación y Desarrollo Ecuador (SENPLADES), la Ley Orgánica de Educación Superior (LOES). Y un incremento sostenido de inversión en recursos humanos, a través de los programas de becas de formación en el exterior y el programa Prometeo; en infraestructura, con planes de inversión en Universidades y escuelas politécnicas.

Palabras Claves: Universidad; tecnología; información; educativo; desarrollo. 


\title{
La educación superior en el Ecuador Vs Las nuevas tecnologías
}

Vol. 1, núm. 5., (2017)

Paulino Javier Suarez Guamán; María Pastora Guamán Sánchez; Virginia Johanna Suarez Guamán; Kerly Pastora Suarez Guamán

\begin{abstract}
The arrival of the Internet to all areas of society has not escaped the educational environment, since many years ago distance education has been incorporated through virtual universities, which are a preamble to what the future environment gives us. information technology and its application in educational environments at the university level and other high-level studies. The methodology used in the present investigation is a non-experimental documentary literature review. In view of the fact that different articles and research works housed in web pages were used as a research method, referring to the subject of higher education in Ecuador and if there have been advances in technology to improve it. Some conclusions that can be reached is that with the change of power in Ecuador in 2007, the theme of new technologies in the higher education environment in Ecuador was institutionalized with the creation of the Higher Education Secretariat, Science, Technology and Innovation (SENASCYT), the National Secretariat of Planning and Development Ecuador (SENPLADES), the Organic Law of Higher Education (LOES). And an increase and sustained investment in human resources, through the programs of training scholarships abroad and the Prometeo program; in infrastructure, with investment plans in Universities and Polytechnics
\end{abstract}

Keywords: University; technology; information; educational; development. 


\section{La educación superior en el Ecuador Vs Las nuevas tecnologías}

Vol. 1, núm. 5., (2017)

Paulino Javier Suarez Guamán; María Pastora Guamán Sánchez; Virginia Johanna Suarez

Guamán; Kerly Pastora Suarez Guamán

\section{Introducción.}

La entrada del nuevo siglo viene acompañada con nuevas fuerzas de cambio en las instituciones de educación superior (IES), en las cuales está impactando una nueva generación de reformas. Estos nuevos fenómenos están asociados a la emergencia de una vigorosa globalización del conocimiento, un mercado educativo sin fronteras, nuevos proveedores y, con ellos, nuevos modelos de ofertas educativas. Esta nueva situación, muy competitiva internacionalmente, puede ser amenazadora para nuestros países si se descuida el calibre de su alcance (Guadilla, 2003).

Desde hace más o menos un cuarto de siglo, según Hess (1997), inició en el terreno educativo la incorporación de las llamadas tecnologías de la información y de la comunicación (TIC), primero en los países industrializados y después en aquéllos con grados menores de desarrollo. De acuerdo con diversas declaraciones de políticos y expertos, en los próximos años esta incorporación no sólo se acrecentará y acelerará, sino será estimulada tanto en las instituciones públicas como en las privadas (Rama, 2006).

La educación vive un tiempo revolucionario, cargado por lo mismo de esperanzas e incertidumbres. Esto se manifiesta con claridad en el acercamiento de la educación a las nuevas tecnologías de la información y la comunicación (NTIC). En torno a ese contacto, existe hoy un verdadero bullir de conceptos e iniciativas, de políticas y prácticas, de asociaciones y organismos, de artículos y libros. Las esperanzas se mezclan con las frustraciones; las utopías, con las realidades. Los gobiernos miden su grado de sintonía con la sociedad de la información en base al número de escuelas conectadas y a la proporción de computadoras por alumnos. Los 


\section{La educación superior en el Ecuador Vs Las nuevas tecnologías}

Vol. 1, núm. 5., (2017)

Paulino Javier Suarez Guamán; María Pastora Guamán Sánchez; Virginia Johanna Suarez Guamán; Kerly Pastora Suarez Guamán

expertos evalúan y critican; los profesores deben adaptarse a exigencias hasta ayer desconocidas;

y los empresarios ofrecen productos, servicios, marcas, experiencias e ilusiones en un mercado educacional cada vez más amplio y dinámico. (Mollis, 2010).

Las temáticas al respecto, giran en torno a adoptar un escenario de cambio que pudiera orientar los esfuerzos de actores, instituciones y gobiernos acerca de nuevas políticas regionales y de Estado en la educación superior a favor de un desarrollo sustentable, desde dos grandes dimensiones: alcanzar la mayor cobertura social con equidad e igualdad; y, el desarrollo de alternativas en la producción y transferencia de conocimientos y aprendizajes (Didriksson et al 2008).

En el contexto latinoamericano, las universidades a distancia han marcado esa evolución hacia las nuevas tecnologías orientadas a la educación superior, casos como México (UNAM, Guadalajara, Veracruz), Argentina (UBA a través de las Facultades y fundamentalmente la de Ciencias Económicas, Quilmes), la Universidad Nacional Autónoma de Honduras y la Universidad Nacional Abierta en Venezuela, son ejemplo de ello.

En el caso ecuatoriano en el año 1976, la Universidad Técnica Particular de Loja, UTPL crea la modalidad abierta e inicia sus labores en octubre de ese mismo año, con el propósito de atender a un amplio sector del Magisterio Nacional (profesorado de secundaria) que no había podido acceder a la educación universitaria. Nace con los institutos de Ciencias Pedagógicas y Ciencias Básicas y la oferta académica de las especialidades de: Pedagogía (940), Matemáticas (251), Física (24) y Química (58); en esta oportunidad se alcanzó una matrícula total de 1273 alumnos. (Torres, 2003). 


\section{La educación superior en el Ecuador Vs Las nuevas tecnologías}

Vol. 1, núm. 5., (2017)

Paulino Javier Suarez Guamán; María Pastora Guamán Sánchez; Virginia Johanna Suarez Guamán; Kerly Pastora Suarez Guamán

Esa misma educación a distancia ha evolucionado y actualmente algunas Universidades la ofrecen con el apoyo de tecnologías, si bien son escasas, se espera un crecimiento significativo a medida en que el Internet incremente su tasa de penetración y conforme las universidades adopten las nuevas formas de hacer educación a distancia de calidad y en la medida en que se proyecten y se adapten a las tendencias de este nuevo siglo, lo que les permitirá seguir vigentes (Torres, 2003).

La Educación superior del país atraviesa un proceso de cambio permanente en que el nivel académico debe estar a la par con las nuevas tecnologías se ha tornado de vital importancia en el proceso de enseñanza aprendizaje. Como está el mundo atiborrado de información hoy en día la realidad académica donde existe una tendencia a cambiar lo que está ya en desuso modificándolo de la mejor forma posible y fortalecer lo que está dando resultados eficientes en conllevar una educación tecnológica a la vanguardia del conocimiento real, virtual y de actualidad. (Castro, 2016)

En el presente trabajo de investigación seguiremos tratando de descubrir si existen otras herramientas que no sea la educación a distancia, que hayan sido empleadas por las universidades ecuatorianas en ese proceso de adecuación a las nuevas tecnologías disponibles hasta el momento. 


\section{La educación superior en el Ecuador Vs Las nuevas tecnologías}

Vol. 1, núm. 5., (2017)

Paulino Javier Suarez Guamán; María Pastora Guamán Sánchez; Virginia Johanna Suarez Guamán; Kerly Pastora Suarez Guamán

\section{Metodología.}

La metodología empleada en la presente investigación es una revisión bibliográfica documental de tipo no experimental. En vista que se utilizó como método de investigación diferentes artículos y trabajos de investigación alojados en páginas web, referente a la temática de la educación superior en el Ecuador y si han habido avances en materia tecnológica para mejorarla.

\section{Resultados}

Figura 1. Países y su Índice de la Economía de Conocimiento

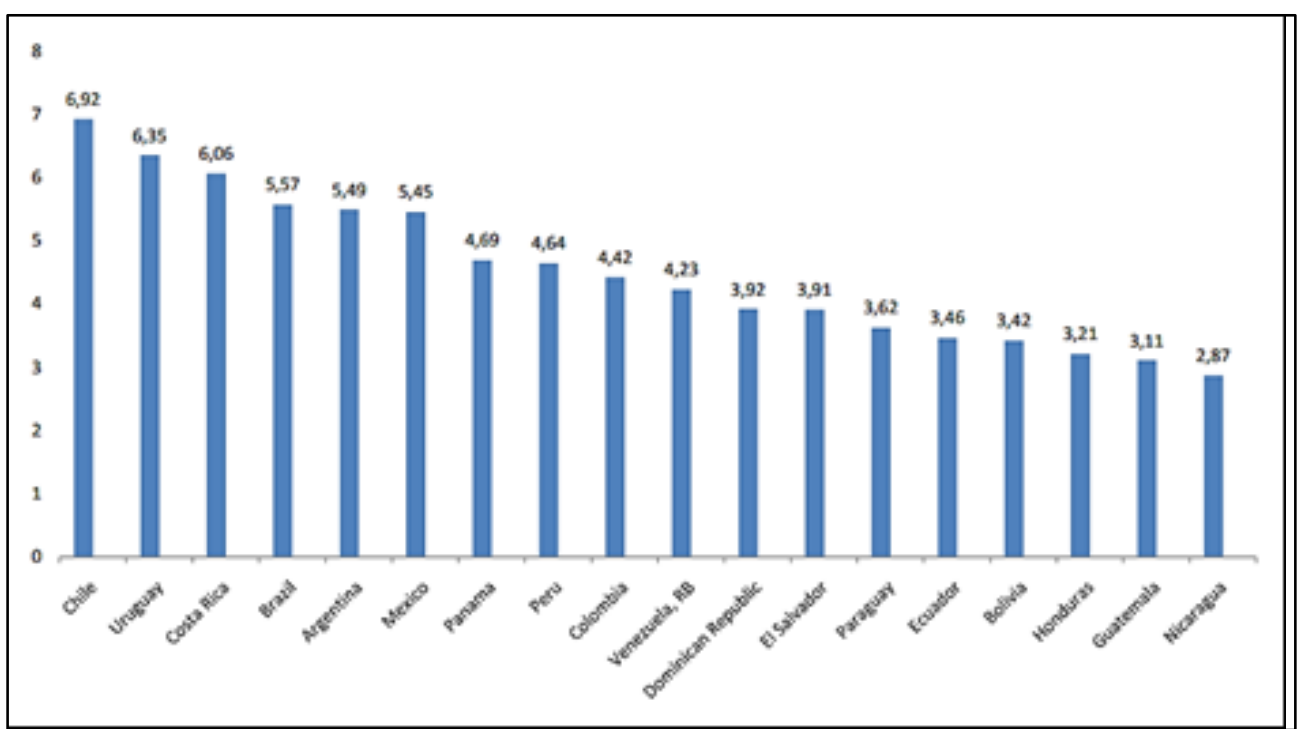

Fuente: The World Bank, Knowledge Assessment Methodology (2009) (Vinueza et al 2017).

- En los índices presentados se puede visualizar que Ecuador está entre los últimos países que tienen acceso a una sociedad de conocimiento, a pesar de que en los artículos 26, 27 y 28 de la 


\section{La educación superior en el Ecuador Vs Las nuevas tecnologías}

Vol. 1, núm. 5., (2017)

Paulino Javier Suarez Guamán; María Pastora Guamán Sánchez; Virginia Johanna Suarez

Guamán; Kerly Pastora Suarez Guamán

Constitución de la República del Ecuador, se establece que la educación es un derecho de cada ciudadano y un deber obligatorio del Estado, además señala que el Estado debe dar prioridad a la educación y por tanto, debe garantizar la igualdad de inclusión para todas las personas y ciudadanos que accedan a ella. Por otro lado, señala que la comunidad educativa en este sentido debe fomentar iniciativas de ayuda hacia la comunidad, desarrollando competencias, habilidades y capacidades en torno a crear nuevos productos tecnológicos reproductores de conocimiento, es decir; lo señalado en estos tres artículos se entiende que la educación debe estar orientada hacia el interés colectivo dejando a un lado los intereses individuales. (Vinueza et al 2017).

- Desde el 2008 a la actualidad, se ha visto una incremental y sostenida inversión en recursos humanos, a través de los programas de becas de formación en el exterior y el programa Prometeo; en infraestructura, con planes de inversión en Universidades y Escuelas politécnicas, junto a construcción de cuatro nuevas universidades en 'áreas estratégicas'; en investigación, con financiamiento a proyectos y programas de investigación, entre los más importantes. Al mismo tiempo, se ha planteado el objetivo de recuperar el rol director del Estado en la educación superior (Asamblea Constituyente, 2008b; CONEA, 2009, p. 1). Así, se han puesto en marcha iniciativas de evaluación y acreditación de las Instituciones de Educación Superior, que han culminado con el cierre de quince de estas por 'falta de calidad' (CEAACES, 2013c) (Milia, 2014).

- Desde la llegada al poder de Rafael Correa, en enero de 2007, se pudieron observar algunos signos de la importancia que el gobierno entrante le daría a la Ciencia y la Tecnología en su 


\section{La educación superior en el Ecuador Vs Las nuevas tecnologías}

Vol. 1, núm. 5., (2017)

Paulino Javier Suarez Guamán; María Pastora Guamán Sánchez; Virginia Johanna Suarez Guamán; Kerly Pastora Suarez Guamán

plataforma de gobierno. En 2007, un documento de planificación específica para el sector planteaba ya áreas estratégicas y establecía como insumo para el 'Desarrollo Humano' la ciencia y la tecnología (SENASCYT, 2007), junto a un plan nacional de desarrollo que se planteaba como quinta meta 'impulsar la investigación, la ciencia y la tecnología' (SENPLADES, 2007, p. 50). Sin embargo, no sería hasta sancionada la nueva constitución que, finalmente, se constituiría un nuevo espacio para el desarrollo de políticas públicas con injerencia sobre las actividades de investigación científica y tecnológica. La constitución sentó las bases y, dos años después, la LOES -junto a su posterior reglamentación, casi un año más tarde- hizo operativos algunos de sus principales principios. (Milia, 2014).

- En la Constitución, también, queda explícito el vínculo de la Ciencia y la Tecnología con la Educación Superior (ES), el artículo 350 establece como funciones de la Educación Superior la formación académica y profesional, pero también la investigación científica y tecnológica, la innovación, y la finalidad de 'construir soluciones' para los problemas del país. Allí, queda claro que el destino de la IES y de la producción de conocimiento está estrechamente vinculado. Al mismo tiempo, la necesidad de que el sistema de ES esté articulado al plan nacional de desarrollo, o Plan Nacional del Buen Vivir, queda clara en el artículo 351. (Milia, 2014).

- En el caso de las instituciones gubernamentales, sobre todo las relacionadas con la educación básica, sus temas de preocupación centrales parecen ser los de equipamiento e infraestructura, capacitación de docentes y establecimiento de redes y portales; esto es, asuntos relativos más al cómo, que con el qué o para qué. Así tenemos, por ejemplo en Ecuador, el proyecto 


\section{La educación superior en el Ecuador Vs Las nuevas tecnologías}

Vol. 1, núm. 5., (2017)

Paulino Javier Suarez Guamán; María Pastora Guamán Sánchez; Virginia Johanna Suarez

Guamán; Kerly Pastora Suarez Guamán

Maestr@s.com para adquisición de equipos y capacitación para profesores; el de Navegar, de formación en informática básica; el establecimiento de la Comisión Nacional de Interconectividad y el portal Educar Ecuador (Ramírez, 2006).

- Las instituciones de educación superior, aunque mantienen la preocupación por la infraestructura y el equipamiento y algunas cuentan con proyectos de producción de materiales y programas, parecen poner mayor atención a los temas de establecimiento de portales y redes así como a los de educación a distancia. Por ejemplo, anotamos los proyectos ecuatorianos RELATED, de la Escuela Superior Politécnica del Litoral, Cyber-ESPE, de la Politécnica del Ejército y el de aulas virtuales, de la Universidad Técnica Particular de Loja (Ramírez, 2006).

\section{Conclusiones.}

- Como decíamos, las capacidades estatales, pero sobre todo las institucionales, en gestión de ciencia, tecnología e innovación se vuelven un punto clave para la utilización estratégica de los instrumentos de política existentes con una importante y sostenida inversión, lo que evidencia una decisión de estado para el financiamiento de estas actividades. Quedan incógnitas planteadas, sobre la incorporación del ecuador a las redes internacionales de producción de ciencia y tecnología, y la sociedad del conocimiento, ¿Qué magnitud alcanzará? ¿Cuáles serán sus características distintivas? ¿Qué impactos generará en el mediano y largo plazo, sobre todo, en la vida universitaria ecuatoriana? ¿Cuándo y cómo se estabilizarán, finalmente, las relaciones de la ciencia, tecnología y la educación superior con la sociedad ecuatoriana? (Milia, 2014). 


\section{La educación superior en el Ecuador Vs Las nuevas tecnologías}

Vol. 1, núm. 5., (2017)

Paulino Javier Suarez Guamán; María Pastora Guamán Sánchez; Virginia Johanna Suarez Guamán; Kerly Pastora Suarez Guamán

- El $72 \%$ de las universidades ecuatorianas ofrecen programas a distancia y semipresenciales y con tendencia creciente, lo que nos lleva a pensar que en algún momento (mediano plazo) todas las universidades o su gran mayoría ofrecerán estudios a distancia y en la medida de sus posibilidades las fortalecerán con componentes virtuales (Torres, 2003).

- Llegamos a la conclusión de que a pesar de que ya la mitad de los ecuatorianos tiene acceso al internet, un poco menos de esta población usa el internet como modo de educación y aprendizaje, la disminución ha sido en respecto al uso del internet, esa pequeña disminución que podemos observar es debido a la falta de enseñanza del Internet y de las TIC como manera de aprendizaje, una debida orientación con respecto al uso del internet la cual tiene una vital importancia el correcto uso (Vinueza et al 2017).

\section{Recomendaciones.}

- En las universidades que son tradicionalmente presenciales se puede notar la necesidad de ir incorporando nuevas TIC, se visibiliza la necesidad de contar con modelos pedagógico más flexibles, centrados en especialmente en los estudiantes, donde ellos lleguen a ser los protagonistas de un nuevo aprendizaje significativo y no solo ser actores invitados que mantiene una posición pasiva frente al reto que representa una educación moderna, propia del siglo XXI en un mundo globalizado (Vinueza et al 2017).

- La presencia de las tecnologías de información y comunicación durante el proceso de enseñanza en las Instituciones de Educación Superior está orientada hacia el desempeño docente y requiere de su aplicación inmediata a través de un Sistema de Estrategias accesibles 


\section{La educación superior en el Ecuador Vs Las nuevas tecnologías}

Vol. 1, núm. 5., (2017)

Paulino Javier Suarez Guamán; María Pastora Guamán Sánchez; Virginia Johanna Suarez

Guamán; Kerly Pastora Suarez Guamán

$\mathrm{y}$ asequibles con el fin de que el estudiante pueda beneficiarse en todo momento de su utilización (Vinueza et al 2017).

- Es necesario aclarar que se debe construir un cambio de carácter social e intelectual como aspecto esencial para comprender y gozar de los procesos tecnológicos y comunicativos entre el estudiante y el entorno virtual (Vinueza et al 2017).

- Los docentes universitarios deben adquirir nuevas tácticas de enseñanza-aprendizaje, que involucren el uso de TIC, ya que son éstas las que ayudan a comprenden las relaciones de los nuevos mecanismos utilizados por grandes universidades para la sistematización del conocimiento por lo que se encuentra al alcance de todos, permitirá a la institución ser más competitiva frente a otras, emitir conocimientos que perduren con el tiempo, y adentrarse a la globalización ya que la tecnología es un idioma universal (Vinueza et al 2017).

\section{Bibliografía.}

Castro, J. (2016). La Educación Superior del Ecuador. Conferencia realizada en el Instituto Tecnológico Bolivariano de Tecnología (ITB). Guayaquil, Ecuador.

Guadilla, C. G. (2003). Balance de la década de los ' 90 y reflexiones sobre las nuevas fuerzas de cambio en la educación superior. Las universidades en América Latina, 17.

Rama, C. (2006). La tercera reforma de la educación superior en América Latina y el Caribe: masificación, regulaciones e internacionalización. Revista Educación y Pedagogía, 18(46), 11-24.

Ramírez Romero, J. L. (2006). Las tecnologías de la información y de la comunicación en la educación en cuatro países latinoamericanos. Revista Mexicana de investigación educativa, 11(28).

Torres, J. C. (2003). Diagnóstico de la Educación Superior Virtual en Ecuador. La educación superior virtual en américa latina y el caribe, 269. 


\section{La educación superior en el Ecuador Vs Las nuevas tecnologías}

Vol. 1, núm. 5., (2017)

Paulino Javier Suarez Guamán; María Pastora Guamán Sánchez; Virginia Johanna Suarez Guamán; Kerly Pastora Suarez Guamán

Milia, M. F. (2014). Marco de Políticas Públicas de Ciencia, Tecnología y Educación Superior en el Ecuador. Nuevos horizontes: dinámicas y condicionamientos para una Investigación Universitaria de cara a la Sociedad. Congreso Iberoamericano de Ciencia, Tecnología, Innovación y Educación, Buenos Aires, Argentina.

Mollis, M. (2010). Las transformaciones de la Educación Superior en América Latina: Identidades en construcción. Revista Educación Superior y Sociedad (ESS) ISSN: 0798$1228,15(1), 11-24$.

Didriksson, A., \& Medina, E. (2008). Contexto global y regional de la educación superior en América Latina y el Caribe. Tendencias de la Educación superior en América Latina y el Caribe, 1, 21-54.

Vinueza, S. F. V., \& Gallardo, V. P. S. (2017). Impacto de las TIC en la Educación Superior en el Ecuador. Revista Publicando, 4 (11), 355-368 